\title{
White Light Generation with Azide Functionalized Polyfluorene Hybridized on Near-UV Light Emitting Diode
}

\author{
Ilkem Ozge Huyal, Tuncay Ozel, Sedat Nizamoglu, Unsal Koldemir, Donus Tuncel, and Hilmi Volkan Demir \\ Department of Physics, Department of Chemistry, Department of Electrical and Electronics Engineering \\ Bilkent University, Ankara, Turkey TR-06800 \\ Email: volkan@bilkent.edu.tr
}

\begin{abstract}
We present white light generation using poly[(9,9-dihexylfluorene)-co-alt-(9,9-bis-(6azidohexyl)fluorene] (PFA) for the first time. Hybridizing PFA on near-UV LED, we demonstrate high color rendering index up to 91.0.

(C)2007 Optical Society of America

OCIS codes: (160.4890) Organic materials; (230.3670) Light-emitting diodes
\end{abstract}

Today wavelength-conversion based LEDs are more commonly used in white light generation when compared to multi-chip LEDs and monolithic LEDs [1,2]. In wavelength conversion technique, different coating materials such as phosphors [2], nanocrystals [3,4], and polymers [5] have been used by integrating on pump LED sources. Although phosphor is currently the most popular wavelength-converting material, polymers offer a number of advantages that may possibly make them the future material of choice for white light generation. Polymers have high solid-state photoluminescence (PL) quantum efficiencies; they are easily processed, molecularly engineered and coated using very low-cost techniques [5]. In this work for the first time, we present white light generation (Fig. 1) using poly[(9,9-dihexylfluorene)-co-alt-(9,9-bis-(6-azidohexyl)fluorene] (PFA) hybridized on near-UV (n-UV) InGaN/GaN LEDs (Fig. 2). We experimentally demonstrate white light generation shown in Fig. 1 (a), with high color rendering indices (CRI) using only a single layer of PFA integrated on n-UV LED, as shown on the C. I. E chromaticity diagram [2] in Fig. 1 (b).

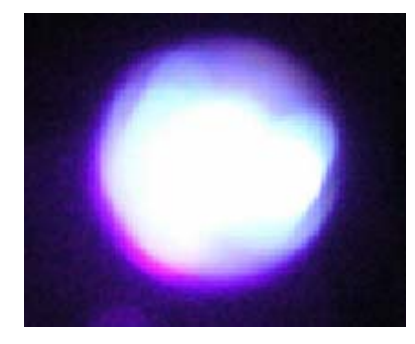

(a)

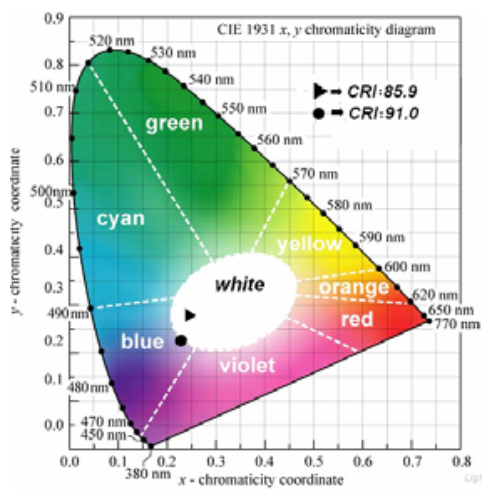

(b)

Figure 1. Our PFA hybridized on n-UV LED: (a) its white light emission and (b) its tristimulus coordinates when the emission is collected from the bottom [ ] and the top [•].
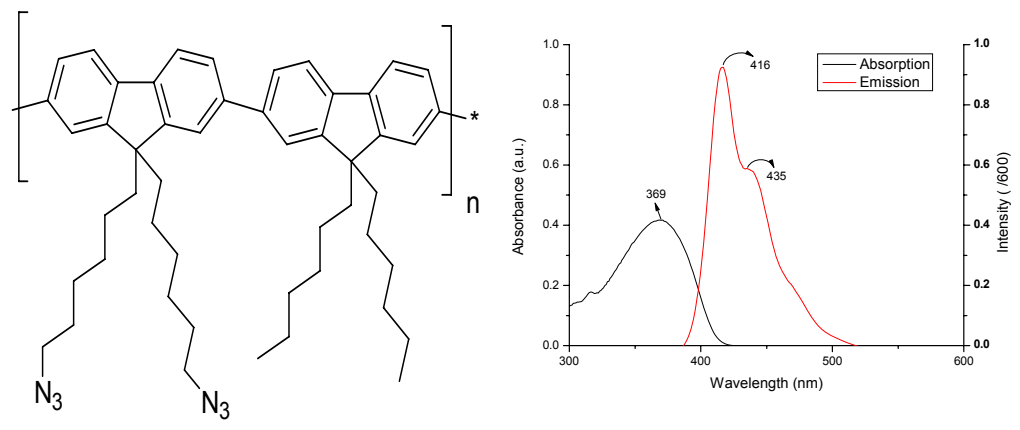

(a)

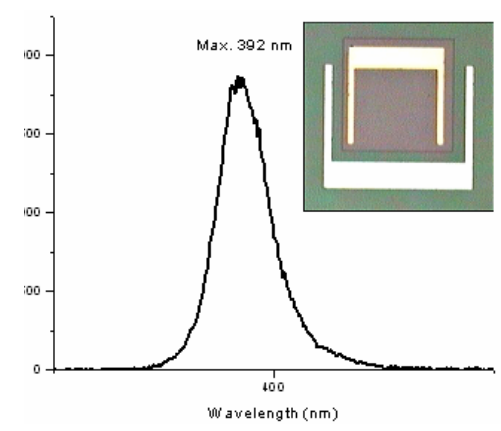

(b)

Figure 2. (a) Condensed formula, absorption and PL spectra of PFA. (b) EL spectrum (onset) and a micrograph (inset) of our n-UVLED.

At Bilkent University Chemistry Laboratory, PFA is synthesized using Suzuki coupling of 2,7-bis[9,9-bis(6bromohexyl)-fluorene and 9,9-dihexylfluorene-2,7-bis(trimethyleneborate) under refluxing in a mixture of $2 \mathrm{M}$ $\mathrm{Na}_{2} \mathrm{CO}_{3}$ /toluene with $\mathrm{Pd}\left(\mathrm{PPh}_{3}\right)_{4}$ as the catalyst [6], followed by excess sodium azide treatment in 


\section{CMO5.pdf}

dimethylformamide (DMF). The synthesized polymer is rigorously purified by precipitating its tetrahydrofuran (THF) solution into methanol and characterized using spectroscopic techniques (including NMR, IR, UV-Vis and fluorescence). The average molecular weight is $55000 \mathrm{Da}$, as measured in THF by GPC. Fig. 2 (a) shows the condensed formula of PFA, along with its optical characterization (absorption and PL spectra measured in solution).

At Bilkent University Nanotechnology Research Center, our InGaN/GaN based n-UV LED is epitaxially grown in a GaN dedicated metal organic chemical vapor deposition (MOCVD) reactor. After the growth of a $25 \mathrm{~nm}$ thick nucleation layer, a $200 \mathrm{~nm}$ thick GaN buffer layer is deposited, which is followed by the Si doped, GaN n layer. Subsequently, 5 quantum wells and barriers with thickness of $\sim 2 \mathrm{~nm}$ and $\sim 3 \mathrm{~nm}$, respectively, are grown as the active region to emit in the $\mathrm{n}-\mathrm{UV}$. A thin $\mathrm{AlGaN}$ hole blocking layer and finally a $\mathrm{Mg}$ doped $\mathrm{p}$ layer is deposited on top of the active layer. $\mathrm{Mg}$ dopants are activated at $750^{\circ} \mathrm{C}$ for 15 minutes. LEDs are then fabricated using standard semiconductor fabrication procedure. P type ohmic contacts are metallized with $\mathrm{Ni}(10 \mathrm{~nm}) / \mathrm{Au}(100 \mathrm{~nm})$ and annealed at $825^{\circ} \mathrm{C}$ for 1 minute in a flowing $\mathrm{N}_{2}$ atmosphere. Also, Ti $(10 \mathrm{~nm}) / \mathrm{Al}(300 \mathrm{~nm})$ are used for the n-type ohmic contacts annealed at $625^{\circ} \mathrm{C}$ for 1 minute in a flowing $\mathrm{N}_{2}$ atmosphere. Fig. 2 (b) shows electroluminescence (EL) of our n-UV LED with its EL peak at $392 \mathrm{~nm}$ and a full width at half maximum of $12 \mathrm{~nm}$, along with its micrograph in the inset.

For hybridization, PFA is dissolved in THF at a concentration of $8.33 \mathrm{mg} / \mathrm{mL}$ and spun at $600 \mathrm{rpm}$ for 10 seconds and $2000 \mathrm{rpm}$ for 30 seconds on the sapphire side of the LED. The film thickness is measured to be $1.5 \mu \mathrm{m}$. Here the n-UV LED platform that is intimately integrated with the PFA thin film serves as an efficient pump source for the excitation of PFA to generate a wide optical spectrum across the visible. The emission spectrum of the PFA film is observed to be broader than that of the PFA in solution. The resulting tristimulus coordinates are experimentally measured to be $(\mathrm{x}=0.22, \mathrm{y}=0.21)$ and $(\mathrm{x}=0.24, \mathrm{y}=0.27)$, with the corresponding high CRIs of 85.9 and 91.0, when the total emission from our PFA-hybridized LED is collected from the bottom and the top, respectively, as shown in Fig. 3 (a) and (b). These tristimulus coordinates fall into the white region of the C.I.E. chromaticity diagram (1931) and these CRIs are high due to the broadening of the emission spectra of the PFA film. Table 1 summarizes the optical properties of our PFA-hybridized LED for the two measurements.

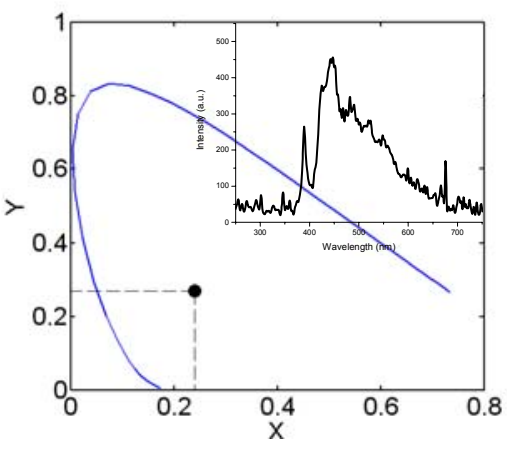

(a)

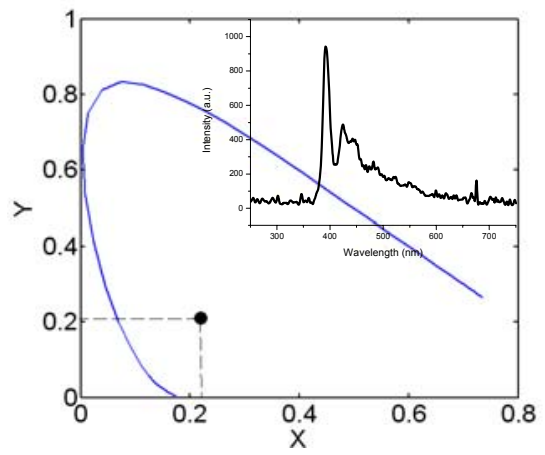

(b)

Figure 3 . (a), (b) Tristimulus coordinates of hybrid PFA LEDs with CRI of 85.9and 91.0 respectively, along with their optical emission spectra in the inset.

Table 1

Chromaticity Coordinates, Color Temperature, and Color Rendering Index

\begin{tabular}{cccc}
$\mathrm{X}$ & $\mathrm{Y}$ & $\mathrm{T}_{c}(\mathrm{~K})$ & $\mathrm{CRI}$ \\
\hline 0.2434 & 0.2723 & 19634 & 85.9 \\
0.2257 & 0.2168 & 34463 & 91.0
\end{tabular}

In conclusion, for the first time, we demonstrated hybrid white LED using PFA as the single coating organic layer integrated on n-UV InGaN/GaN LED and obtained high color rendering indices with such PFA-hybridized LED. We are currently investigating the photostability issues and the mechanisms that broaden the emission spectra of the PFA film.

\section{Acknowledgments}

This work is supported by EU-MC-IRG MOON 021391 and EU-NOE-PHOREMOST 511616 and TUBITAK 104E114, 106E020, 105E065, and 105E066. H.V.D. and S.N. also acknowledge additional support from Turkish Academy of Sciences and TUBITAK.

\section{References}

[1] M. Yamada, et al., IEICE Trans. Electron., E88-C, (9), 1860-1871, (2005)

[2] E. F. Schubert, "Light-Emitting Diodes" (Cambridge University Press, 2006).

[3] S. Nizamoglu, T. Ozel, E. Sari, and H. V. Demir, "White Light Generation with CdSe/ZnS Core-Shell Nanocrystals and InGaN/GaN Light Emitting Diodes,"

Proc. of IEEE Conference on Optoelectronic and Microelectronic Materials and Devices, Perth, Western Australia (2006).

[4] H. Chen, et al., IEEE Photon. Technol. Lett., 18, (13), 1430-1432, (2006).

[5] G. Heliotis, et al, J. Opt. A: Pure Appl. Opt. 8 Appl. Phys. Lett. (87), 103 505, (2006).

[6] Bin Liu, et. al., J. Am. Chem. Soc., 128, 1188-1196, (2006). 